\title{
CORRECTION SYSTEMS UPGRADE FOR THE SNS RING*
}

\author{
Y. Papaphilippou ${ }^{\dagger}$, C.J. Gardner, Y.Y. Lee and J. Wei \\ Brookhaven National Laboratory, UPTON NY 11973, USA
}

\begin{abstract}
In view of the changes in the design of the SNS ring from the original FODO lattice [1] to the 220m hybrid lattice [2] and finally $1.3 \mathrm{GeV}$ compatible $248 \mathrm{~m}$ ring [3], complementary studies have been undertaken, in order to upgrade its correction packages. We review the evolution of the correction systems and present the accelerator physics studies for the adopted schemes and powering plan.
\end{abstract}

\section{THE SNS CORRECTOR PACKAGES}

The latest design of SNS accumulator lattice is of a hybrid type with four identical arcs and four special function straight sections. The arc has a FODO structure, with four cells (8 quadrupoles and dipoles) plus a quadrupole that matches the arcs with the straight sections. The latter contains two quadrupole doublets, setting the total number of quadrupoles at 52. The magnetic elements are placed and powered in a way to preserve a four-fold symmetry. The space between the quadrupoles and dipoles is occupied by low-field correctors and chromaticity sextupoles. They are adjacent to the quadrupoles (upstream or downstream) in order to take advantage of the horizontal or vertical beta function maximum in these locations of the ring [4]. A schematic presentation of one super-period is presented in Fig. 1. A summary of the correction packages for the ring is described in Table 1. In the following paragraphs, we describe each system in more details.

\subsection{Dipole correctors}

The 44 dipole correctors of the SNS ring are represented in Fig. 1 as brown rectangles. Due to the aperture change in the middle of the arc there are two types: 28 of them have a $27 \mathrm{~cm}$ gap (27CDM30 in the magnet nomenclature) and 8 are designed wider, with a $36 \mathrm{~cm}$ gap (36CD30 corresponding to DHCX4 and DHCX6). The remaining 8 correctors (41CD30 or DHVCX10 and DHVCX13) are located in the straight sections, adjacent to the doublets (upstream and downstream) and their gap is even larger $(41 \mathrm{~cm})$. Correctors in the arc produce either a horizontal or a vertical dipole kick and the determination of their positioning is imposed by the FODO structure. The dipole correctors in the straight sections are designed as combined function magnets able to produce both horizontal and vertical dipole kicks. This will enable the necessary beam steering to any direction in these areas, where the beam envelope becomes wider and the closed orbit control is critical for injection, collimation, extraction and the RF system.

\footnotetext{
${ }^{*}$ Work supported by the US Department of Energy

† papap@bnl.gov
}

The perturbation on the closed orbit can be attributed to random quadrupole misalignments and dipole displacements (around $0.5 \mathrm{~mm}$ ), variation of the dipole field (of the order of a few $10^{-4}$ ), or random tilt of the dipoles (of about $0.5 \mathrm{mrad}$ ) [4]. In order to achieve this correction, the dipole correctors are powered independently. In the arc they give an integrated dipole field of around $6.7 \times 10^{-4} \mathrm{~T}$ m which gives the capability of $1.2 \mathrm{mrad}$ kicks, at $1 \mathrm{GeV}$. In the straight sections, they are able to produce approximately twice this integrated field and kick, both in the horizontal and vertical direction. The specified field can be given by running the power supplies at $11 \mathrm{~A}$.

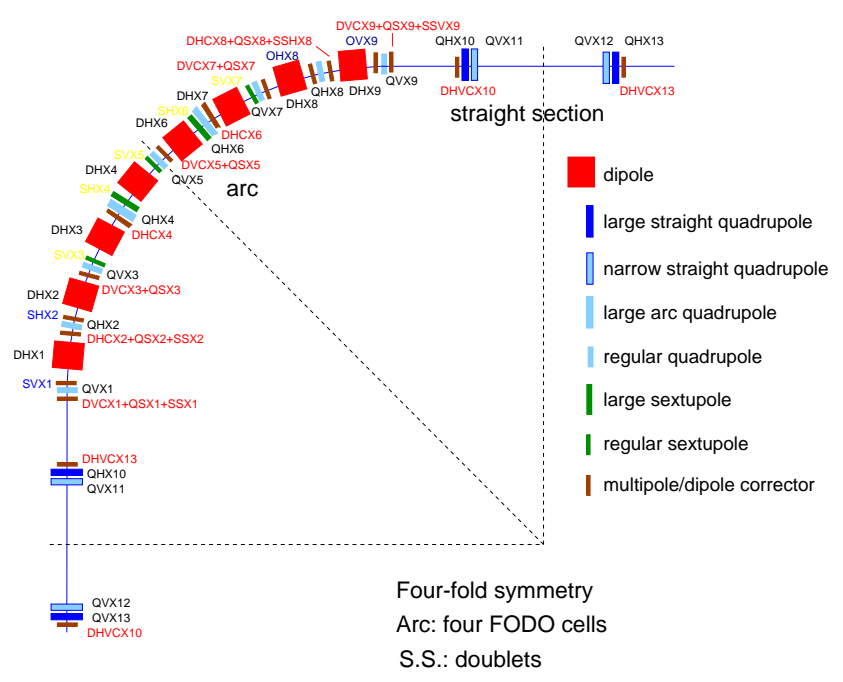

Figure 1: Schematic layout showing dipole, quadrupole, sextupole and correctors of one lattice super-period.

\subsection{Quadrupole correction}

The preservation of the lattice super-periodicity is essential for the good performance of high-intensity rings. Based on first order perturbation theory, a lattice with perfect symmetry does not allow the excitation of resonances other than "structural", i.e. $k_{x} Q_{x}+k_{y} Q_{y}=m N$ where $m$ is the super-periodicity ( 4 for the SNS ring). When the super-periodicity is broken, e.g. by random errors in the magnets, "non-structural" resonances can be excited as well. The combination of lattice perturbation in the presence of large space-charge forces leads to excessive beam loss, as already observed and analyzed theoretically in the KEK PS [5]. The direct observable for a broken superperiodicity is the distortion of the linear optics functions. In the case of the SNS, random and systematic errors in the quadrupole strengths can create significant beta beating and dispersion distortion. Another perturbation comes from the injection chicane and bump which break the four- 
Table 1: Summary of corrector elements for the ring system

\begin{tabular}{lll}
\hline Magnet & Mechanical Nomenclature & Number \\
\hline $\begin{array}{l}\text { Horizontal or Vertical Dipole + Skew Quadrupole + Skew Sex-- } \\
\text { tupole }(\operatorname{arc})\end{array}$ & 28 \\
\hline Horizontal Dipole $($ arc wide aperture) & 36 CD30 & 8 \\
Horizontal and Vertical Dipole (straight section + linac dump) & 41CD30 & $8+1$ \\
\hline Quadrupole TRIM Winding & 21 QT40, 26QT38, 30QT58, 30QT44 & 52 \\
Sextupole & 21 CS26 & 8 \\
Octupole & 21 CO26 & 8 \\
\hline Total number of elements & & 121 \\
Total number of functions & & 178 \\
\hline
\end{tabular}

fold symmetry of the lattice and introduce additional beta wave [6]. In order to compensate these effects and allow a fine tuning of the SNS lattice, every magnet is equipped with TRIM windings able to produce $1 \%$ of the magnet quadrupole strength. The quadrupole gradient produced by the TRIMs is of the order of $5 \times 10^{-2} \mathrm{~T} / \mathrm{m}$, for $1 \mathrm{GeV}$ operation.

Since the early stage of the project, correction schemes have been proposed for the linear optics distortions and quadrupole resonance compensation, by connecting appropriately the quadrupole windings [4]. The total number of quadrupole string families proposed for the original FODO lattice was 7 . With the evolution to the $228 \mathrm{~m}$ hybrid lattice and later to the $248 \mathrm{~m} 1.3 \mathrm{GeV}$ compatible ring, it became apparent that the number of families will be not enough for correcting all optics distortion. An individual powering scheme was proposed to give flexibility in the correction schemes, to assist the orbit correction in the early commissioning stage and to allow the beam-based alignment of the BPMs. Due to the budget considerations of the SNS project, we presently propose a more restrictive correction scheme using 16 families of TRIM strings. In order to achieve the required $1 \%$ quadrupole gradient in each winding, for all five different types of quadrupoles, the power supplies should be able to give currents of 15 to $30 \mathrm{~A}$.

\subsection{Skew quadrupole correction}

The skew quadrupole correctors are used for the linear decoupling of beam motion. Coupling can be mainly attributed to random rolls in the quadrupoles of the SNS ring lattice, whose rms values can be as large as $1 \mathrm{mrad}$. A smaller contribution comes from random and systematic skew quadrupole errors in the magnetic elements. Apart from the linear optics perturbations, skew quadrupole errors can excite the coupling resonances $Q_{x} \pm Q_{y}=N$. Especially for the nominal working point $\left(Q_{x}, Q_{y}\right)=$ $(6.3,5.8)$ which was close to the structural coupling resonance $Q_{x}+Q_{y}=12$, a very demanding correction has to be applied in order to compensate the effect of random quadrupole rolls [7]. In a quite similar way as for quadrupole errors, the large space-charge tune-shift pushes the particles in the resonance and the result is a quite steep increase of beam loss to the $10 \%$ level. It was also shown that the global coupling correction would not be enough to cancel the effect and a local coupling scheme should be employed. In the SNS ring there are no individual skew quadrupole magnets, but on the cores of the arc dipole correctors (27CDM30) separate windings are mounted that can create a skew quadrupole integrated gradient of about $2.25 \times 10^{-2} \mathrm{~T}$ at $10.9 \mathrm{~A}$. They are denoted as QSX[1-9] in Fig. 1. In the baseline scheme, it was foreseen to power only 16 of these windings, i.e the ones at the beginning and the end of the arcs. Due to the results mentioned above [7], the final decision is to power the skew quadrupole windings in every $27 \mathrm{CDM} 30$ correctors, which gives a total of 28 . In order to achieve the required skew quadrupole gradient in each winding the power supplies give currents of $11 \mathrm{~A}$, for $1 \mathrm{GeV}$ operation.

\subsection{Skew-sextupole correction}

The excitation of skew sextupole resonances $2 Q_{x} \pm$ $Q_{y}=N$ and $3 Q_{y}=N$ was found to be one of the most important limitation of the AGS booster. These resonances can be excited by skew sextupole errors coming from rolls in the main dipoles or other geometrical errors in the magnet elements. In order to correct this effect skew sextupole windings are mounted on the 27CDM30 correctors along with the main dipole coils and the skew quadrupole components. Only the one at the beginning an the end of the arc are powered (SSX [8-9]) and provide with an integrated gradient is $6.6 \times 10^{-2} \mathrm{~T} / \mathrm{m}$ (as for the AGS booster). In the initial scheme, the skew sextupole were powered in 2 families. This would have not been enough for correcting any skew sextupole resonance without changing the connection between correctors and would necessitate power supplies producing higher voltage and power with an increased cost. Thus, we require the additional powering of the skew sextupole windings in the 27CDM30 correctors at the beginning of the arc (SSX[1-2]) and the connection of the correctors in 8 families of two, following the arc symmetry. In order to achieve the required skew sextupole field in each corrector, the power supplies should be able to give currents of $13 \mathrm{~A}$, for $1 \mathrm{GeV}$ operation.

\subsection{Sextupole correctors}

The main functionality of the sextupole correctors is the correction of sextupole resonances $3 Q_{x}=N$ and $Q_{x} \pm 2 Q_{y}=N$, excited by sextupole errors in the dipoles, dipole fringe-fields at leading order and the small nonlinear effect of the chromaticity sextupoles [8]. The 8 sextupole correctors are located at the beginning of the arc in 
Table 2: Summary of low field corrector powering for the ring system.

\begin{tabular}{|c|c|c|c|c|}
\hline Element & Original & New & Increase & Justification \\
\hline Dipole & 52 & 54 & 2 & Injection dump dipoles \\
\hline Quadrupole TRIM & 7 & 16 & 9 & $\begin{array}{l}\text { Large beta-beating due to lattice symmetry breaking (injection chi- } \\
\text { cane/bump, quadrupole transfer function, quadrupole errors) }\end{array}$ \\
\hline Skew Quadrupole & 16 & 28 & 12 & Local tilt correction \\
\hline Skew Sextupole & 2 & 8 & 6 & $\begin{array}{l}\text { Skew sextupole resonance correction for odd-even harmonics } \\
\text { (AGS booster) }\end{array}$ \\
\hline Sextupole & 2 & 8 & 6 & $\begin{array}{l}\text { Sextupole resonance correction for odd-even harmonics due to } \\
\text { sext. errors and octupole feed-down }\end{array}$ \\
\hline Octupole & 2 & 8 & 6 & $\begin{array}{l}\text { Octupole resonance correction for odd-even harmonics due to } \\
\text { quad. fringe fields }\end{array}$ \\
\hline Total & 81 & 122 & 41 & $\begin{array}{l}\text { Changes reflect the evolution of the design from the FODO lattice } \\
\text { to the hybrid and the latest } 248 \mathrm{~m} \text { ring }\end{array}$ \\
\hline
\end{tabular}

non-dispersive areas in order to avoid "feed-downs" due to closed-orbit displacements. The correctors are also taking advantage of the high horizontal or vertical beta maximum (SVX1 and SHX1 in Fig. 1). The integrated sextupole gradient produced is about $2.6 \mathrm{~T} / \mathrm{m}$. For the same reason as for the skew sextupole correctors, it was decided to proceed with an individual powering scheme, with 8 power supplies instead of 2 as it was initially proposed. It will allow the possibility of correction of all resonant lines independently of the selected working point [8]. In order to achieve the required sextupole field in each corrector, the power supplies should be able to give currents of $8.4 \mathrm{~A}$, for $1 \mathrm{GeV}$ operation.

\subsection{Octupole correctors}

The main "octupole-like" contribution comes from the leading order quadrupole fringe-fields, which are producing a tune-shift of around 0.025 at $480 \pi \mathrm{mm}$ mrad [9], the third most important contribution after space-charge and chromaticity. The octupole correctors are located at the end of the arc in non-dispersive areas in order to avoid sextupole "feed-downs" due to closed orbit displacements. They are 8 in total and, as for the other correctors, their position is determined by the maxima of the beta functions near the arc focusing and defocusing quadrupoles (OHX1 and OVX1 in Fig. 1). In order to eliminate completely the large tune-shift coming from quadrupole fringe-fields, a third octupole family is necessary in areas where the horizontal and vertical beta functions are approximately equal. Unfortunately, this is not possible to a first stage due to the tight space of the ring in these areas. On the other hand, it would be impossible to correct all octupole type resonances $4 Q_{x}=N, 2 Q_{x} \pm 2 Q_{y}=N$ and $4 Q_{y}=N$ with the initial two-family scheme. For this reason, we proposed individual powering, as for the other non-linear correctors. The integrated octupole gradient given by the correctors is $2.9 \mathrm{~T} / \mathrm{m}^{2}$, which will enhance the efficiency of resonance corrections. In order to achieve the required octupole field in each corrector, the power supplies should be able to give currents of $8.4 \mathrm{~A}$, for $1 \mathrm{GeV}$ operation. These power supplies although increased in number need a factor of two less voltage then the initial ones.

\section{SUMMARY}

The SNS system has evolved considerably since the initial FODO lattice to the hybrid lattice of $228 \mathrm{~m}$ and finally to the $1.3 \mathrm{GeV}$ compatible $248 \mathrm{~m}$ ring. The powering of the correction system had to be reviewed following this evolution. In the original baseline scheme, the total number of power supplies was 81 for the ring. In the new proposal, the total number of ring power supplies is 122 . The benefits of the increase for each correction scheme individually are stated in Table 2. The present powering capability enables the application of the necessary correction schemes independently of the working point choice. This is instrumental for a high intensity machine with very stringent loss level requirements. In addition, due to the individual powering of most of the components the power supplies proposed have much smaller voltage and power requirements and less cables are needed. Finally, as most of the power supplies are of the same kind with maximum current and voltage specs of $20 \mathrm{~A}$ and 35 Volts, they can be possibly interchanged if necessary. The present number of power supplies reflects the absolute minimum needed, as this number will not provide the ability to achieve the high demands of the anticipated $1.3 \mathrm{GeV}$ operation. It is debated that more power supplies are needed for independent control of the quadrupole TRIM windings and more skew quadrupole components for local decoupling schemes.

\section{REFERENCES}

[1] W.T. Weng, et al., PAC'97, Vancouver, B.C., Canada, 1997; Y.Y. Lee, BNL/SNS Tech. Note 26, 1997;

[2] J. Wei, et al., BNL/SNS Tech. Note 66, 2000; PRST-AB, 3:080101, 2000.

[3] J. Wei, et al., BNL/SNS Tech. Note 76, 2000.

[4] C.J. Gardner, BNL/SNS Tech. Note 40, 1997; BNL/SNS Tech. Note 31, 1997; BNL/SNS Tech. Note 82, 2000; BNL/SNS Tech. Note 37, 1997.

[5] S. Mashida, Y. Shoji, AIP Conf.Proc., 377, 160, 1995; Y. Shoji, H. Sato, NIMPR, 399, 5, 1997.

[6] J. Holmes, et al., these proceedings.

[7] A.V. Fedotov, these proceedings.

[8] N. Tsoupas, et al., EPAC 2000, Vienna, 2000.

[9] Y. Papaphilippou, D.T. Abell, EPAC 2000, Vienna, 2000. 